\title{
Clinical characteristics of children with juvenile dermatomyositis recruited within the first 7 months of the CARRAnet registry
}

\author{
Mark F Hoeltzel ${ }^{1 *}$, Mara L Becker ${ }^{1}$, Angela B Robinson ${ }^{4}$, Brian M Feldman 5 , Adam Huber ${ }^{2}$, Ann M Reed ${ }^{3}$, \\ Juvenile Myositis CARRA Subgroup ${ }^{6}$, CARRAnet Investigators ${ }^{6}$
}

From 2011 Pediatric Rheumatology Symposium sponsored by the American College of Rheumatology Miami, FL, USA. 2-5 June 2011

\section{Purpose}

Performing quality clinical and translational research in juvenile dermatomyositis (JDM) is difficult due to the rarity of the disease. The Childhood Arthritis and Rheumatology Research Alliance (CARRA) initiated a multicenter observational cohort study to create a foundational clinical database for the major rheumatic diseases of childhood, including JDM. Initial data from the JDM cohort (prevalent and incident cases) enrolled in the first 7 months of this ongoing study are described here.

\section{Methods}

Children under the age of 21 with onset of JDM prior to 16 yrs old were included. JDM was established using modified Bohan and Peter criteria. Subjects or their guardians were consented, and clinical data were collected from the patients/guardians and medical providers using both general and JDM-specific case report forms at the time of enrollment. Data regarding demographics, disease characteristics, diagnostic assessment, and medication exposure were collected. Baseline measures of muscle strength, physical functioning, and quality of life were performed, including the Childhood Myositis Assessment Scale (CMAS), Childhood Health Assessment Questionnaire (CHAQ), Health Related Quality of Life measure (HRQOL), ACR Functional Class rating, global disease assessments, and pain scores. Data were pooled and stored in a secure centralized database and de-identified prior to analysis. IRB approval was obtained at each enrolling site.

\section{Results}

Between May 28, 2010 and December 28, 2010, 102 subjects meeting modified Bohan and Peter criteria for JDM were enrolled from 23 sites in the U.S. The average number of patients enrolled per site was 5.3 (range 1-18). A summary of subject demographics and disease characteristics is provided in Table 1. Median (quartiles) CMAS score at enrollment was $50(45,52)$ with a range from 0 52. Median CHAQ score at enrollment was $0(0,0.5)$ with a range from $0-3$. Median physician and subject global assessment scores at enrollment were $1(0,2)$ with a range from $0-8$, and $1(0,4)$ with a range from $0-9$ respectively. Subject-reported median pain score at enrollment was 1 $(0,2)$ with a range from $0-8$. HRQOL and ACR functional class results are represented in Figure 1.

\section{Conclusion}

In 7 months, the ongoing CARRA registry of children with rheumatic diseases has collected clinical data on 102 children with JDM and has the potential to become one of the largest JDM cohorts in the world. This registry provides the infrastructure needed to advance clini$\mathrm{cal}$ and translational research and represents a major step towards improving outcomes of children with JDM.

\section{Disclosure}

Mark F. Hoeltzel: None; Mara L. Becker: None; Angela B. Robinson: None; Brian M. Feldman: None; Adam Huber: None; Ann M. Reed: None; Juvenile Myositis CARRA Subgroup: None; CARRAnet Investigators: None.

${ }^{1}$ Children's Mercy Hospital, Kansas City, MO, USA

Full list of author information is available at the end of the article

(c) 2012 Hoeltzel et al; licensee BioMed Central Ltd. This is an Open Access article distributed under the terms of the Creative Commons Attribution License (http://creativecommons.org/licenses/by/2.0), which permits unrestricted use, distribution, and reproduction in any medium, provided the original work is properly cited. 
Table 1 Demographics and disease characteristics

\begin{tabular}{|c|c|c|c|c|c|}
\hline Characteristc & Number (\%) of subjects & & Characteristic & Number (\%) with characteristic ever & Number (\%) with characteristic at enrollment \\
\hline Sex & & & Elevated muscle enzymes & $86 / 89(97 \%)$ & $6 / 89(7 \%)$ \\
\hline Female & $80(78 \%)$ & & Arthritis & $34 / 86(40 \%)$ & $4 / 86(5 \%)$ \\
\hline Male & $22(22 \%)$ & & Calcinosis & 10/87 (12\%) & $7 / 87(8 \%)$ \\
\hline Ratio Female:male & $3.6: 1$ & & Cardiac involvement & $1 / 85(1 \%)$ & $1 / 85(1 \%)$ \\
\hline Race/Ethnicity & & & GI Ulceration & $3 / 85(4 \%)$ & $1 / 85(1 \%)$ \\
\hline White, Non-Hispanic & $65(64 \%)$ & & Dysphagia/Dysphonia & 26/84 (31\%) & $1 / 84(1 \%)$ \\
\hline White, Hispanic & $13(13 \%)$ & & ILD & $1 / 84(1 \%)$ & $0 / 84(0 \%)$ \\
\hline African American & $9(9 \%)$ & & Muscle weakness: & & \\
\hline American Indian & $1(1 \%)$ & & None & - & $62 / 91(68 \%)$ \\
\hline Pacific Islander & $1(1 \%)$ & & Mild & - & 23/91 (25\%) \\
\hline Multi-racial & $10(10 \%)$ & & Moderate & - & 4/91 (4\%) \\
\hline Unknown & $3(3 \%)$ & & Severe & - & $2 / 91(2 \%)$ \\
\hline $1^{\circ}$ Family hx of autoimmunity & 21/92 (23\%) & & Periungual telangiectasia & - & $39 / 86(45 \%)$ \\
\hline Positive ANA & $55 / 85(65 \%)$ & & Contractures & - & 7/91 (8\%) \\
\hline Chronology & Median, yrs (quartiles) & Range, yrs & V or shawl sign & - & $5 / 91(6 \%)$ \\
\hline Age & $10.6(7.1,14.8)$ & $2.4-20.8$ & Lipodystrophy & - & 4/91 (4\%) \\
\hline Age of onset & $6.0(3.3,9.5)$ & $0.9-15.9$ & Skin ulcer & - & $3 / 91(3 \%)$ \\
\hline Disease duration & $3.0(1.7,5.7)$ & $0.2-12.8$ & Malar or facial erythema & - & $24 / 90(27 \%)$ \\
\hline Time to rhematologie care & $0.32(0.13,0.67)$ & $0-5.1$ & Gottron sign, papules, or heliotrope & - & 44/91 (48\%) \\
\hline
\end{tabular}




\section{Figure 1: A. Worst Ever ACR Functional Class}

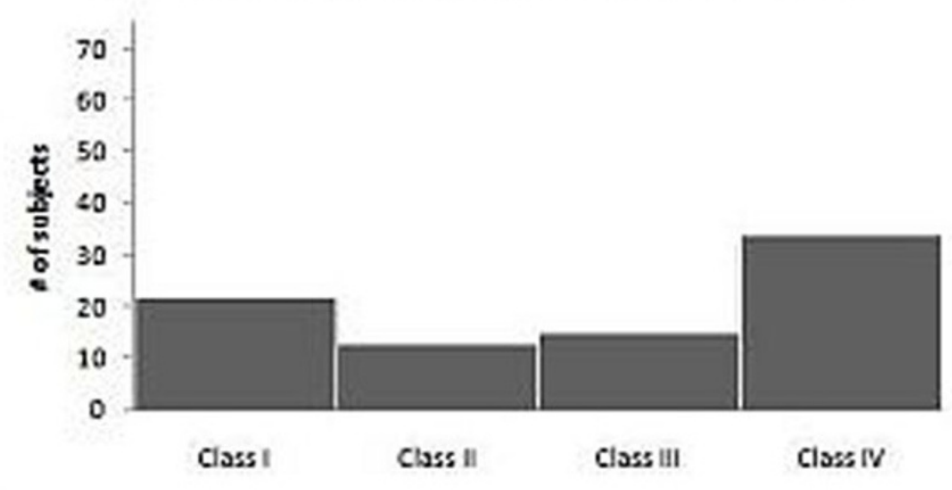

\section{B. Current ACR Functional Class}

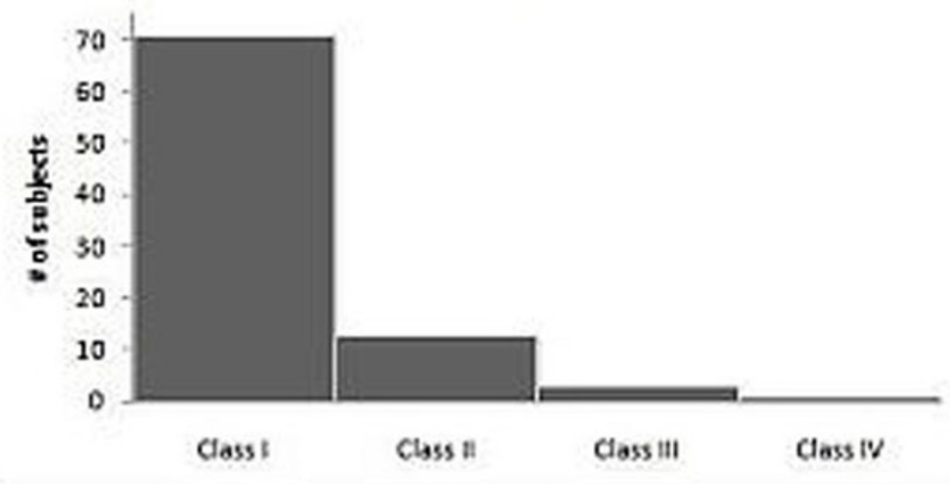

\section{Current HRQOL}

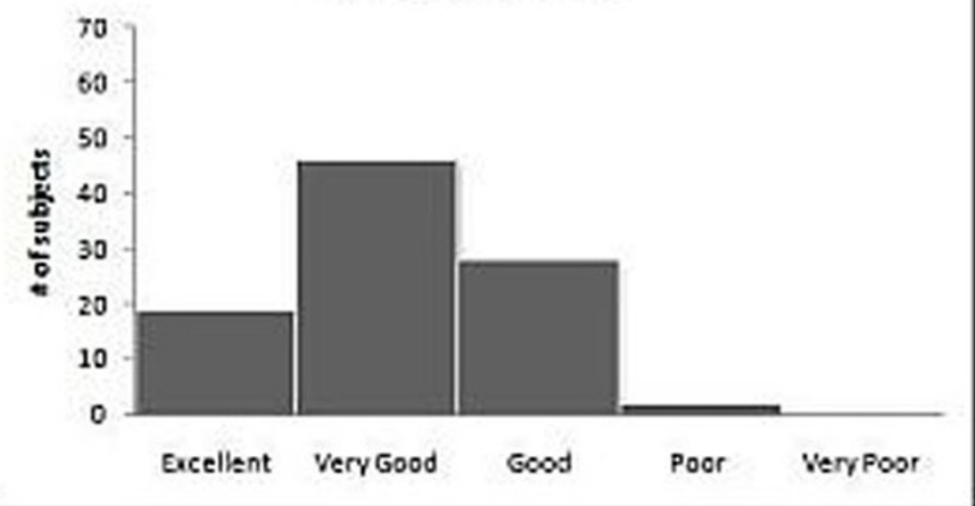

Figure 1

\section{Author details}

${ }^{1}$ Children's Mercy Hospital, Kansas City, MO, USA. ${ }^{2}$ IWK Health Centre, Halifax, NS, Canada. ${ }^{3}$ Mayo Clinic, Rochester, MN, USA. ${ }^{4}$ Rainbow Babies and Childrens Hospital, Cleveland, OH, USA. ${ }^{5}$ The Hospital for Sick Children, Toronto, ON, Canada. ${ }^{6}$ CARRA, Stanford, CA, USA.
doi:10.1186/1546-0096-10-S1-A12

Cite this article as: Hoeltzel et al:: Clinical characteristics of children with juvenile dermatomyositis recruited within the first 7 months of the CARRAnet registry. Pediatric Rheumatology 2012 10(Suppl 1):A12.

Published: 13 July 2012 